University of New Hampshire

University of New Hampshire Scholars' Repository

10-1994

\title{
Viewing a Graph in a Virtual Reality Display is Three Times as Good as a 2D Diagram
}

\author{
Colin Ware \\ University of New Hampshire, Durham, colin.ware@unh.edu \\ Glenn Franck \\ University of New Brunswick
}

Follow this and additional works at: https://scholars.unh.edu/ccom

Part of the Computer Sciences Commons, and the Oceanography and Atmospheric Sciences and Meteorology Commons

\section{Recommended Citation}

Ware, C.; Franck, G., "Viewing a graph in a virtual reality display is three times as good as a 2D diagram," in Visual Languages, 1994. Proceedings., IEEE Symposium on, pp.182-183, 4-7 Oct 1994 doi: 10.1109/ VL.1994.363621

This Conference Proceeding is brought to you for free and open access by the Center for Coastal and Ocean Mapping at University of New Hampshire Scholars' Repository. It has been accepted for inclusion in Center for Coastal and Ocean Mapping by an authorized administrator of University of New Hampshire Scholars' Repository. For more information, please contact Scholarly.Communication@unh.edu. 


\title{
Viewing a Graph in a Virtual Reality Display is Three Times as Good as a 2D Diagram
}

\author{
Colin Ware and Glenn Franck
}

Faculty of Computer Science, University of New Brunswick

Box 4400, Fredericton, NB, Canada E3B 5A3, cware@unb.ca

\begin{abstract}
An experiment is reported which tests whether network information is more effectively displayed in a three dimensional space than in a two dimensional space. The experimental task is to trace a path in a network and the experiment is carried out in $2 D$, in a $3 D$ stereo view, in a $3 D$ view with head coupled perspective, and in a $3 D$ stereo view with head coupled perspective; this last condition creates a localized virtual reality display. The results show that the motion parallax obtained from the head coupling of perspective is more important than stereopsis in revealing structural information. Overall the results show that three times as much information can be perceived in the head coupled stereo view as in the $2 D$ view.
\end{abstract}

\section{Introduction}

As the display of three dimensional rather than two dimensional information becomes commonplace due to advances in computer graphics hardware, the development of visual languages and symbologies that work in 3D will become increasingly important. One of the outstanding issues is the question of whether there is any advantage to creating visual languages that are truly three dimensional since the information is typically not spatial in nature. The present study addresses this issue by empirically testing the comprehension of network information shown in $3 \mathrm{D}$ and in $2 \mathrm{D}$.

A useful and interesting method for examining three dimensional structures is to couple a perspective stereoscopic view of a 3D scene to the user's eye positions and update the view in real-time as the user moves. The key elements of this are a high resolution monitor capable of running at a high frame rate, stereo glasses and some method for tracking the user's head position $[1,2]$. The position of the user's two eyes are computed from the head position and separate images are generated showing the correct perspective view of a set of virtual objects somewhere in the vicinity of the monitor screen. The result is a localized "Virtual Reality" (VR) environment which has a number of advantages over the much talked about immersive virtual reality, not the least of these being that the everyday workspace of desk, filing cabinet, co-workers and coffee mug are not excluded. In our previous work we have called this "Fish Tank VR" to characterize its localized nature and distinguish it from the full immersion kind [4].

\section{Experiment}

While it is clear that the kind of 3D display described above has advantages for people who wish to look at representations of $3 \mathrm{D}$ data, such as images of bones used for planning orthopaedic surgery, it is not clear that abstract data can benefit from $3 \mathrm{D}$ representation. The key question is, is a $3 \mathrm{D}$ diagram better than a $2 \mathrm{D}$ diagram? Some previous studies have suggested that there is and advantage but say nothing about how large the advantage is $[1,3]$.

The purpose of this experiment was to determine how much more, or less, can be perceived in a head coupled stereo display used to display network information. On a given trial the subject viewed a randomly laid out network of nodes and arcs with two nodes highlighted. The task was to say if there was a path between the the highlighted nodes, while in fact there was either a path of length two or no path, each occurring $50 \%$ of the time. There were four viewing modes.

1) 2D: no stereo, no rotation; the $3 \mathrm{D}$ graph was projected onto a 2D plane using an orthographic (parallel) projection by removing $\mathrm{Z}$ axis information, hence no overlap information was available.

2) Stereo perspective: no rotation; this condition made use of a pair of StereoGraphics CrystalEyes LCD shutter glasses to provide the disparity depth cues.

3) Head coupled perspective: the scene's perspective projection changed continuously according to the subject's measured head position; the perspective projection was defined by a single viewpoint centered between the eyes.

4) Stereo, head coupled perspective: same as above, except with stereo; the correct view was generated for each eye position (continuously updated).

The numbers of nodes used in the different conditions were as follows; these had been established as useful ranges in a previous pilot study. 
1) $21,42,63,84,105$

2) $51,81,111,141,171$

3) $81,117,153,189,225$

4) $111,156,201,249,291$

The number of arcs was the number of nodes multiply by $4 / 3$.

This experiment involved 11 participants. (The other procedure details are given in a technical report [5].)

\section{Results and discussion}

Figure 1 summarizes the error data from this experiment. This figure shows a sequence of curves with varying gradients which appear to be roughly multipliers of each other with respect to the graph size. That is, error rate appears to be directly proportional to the number of nodes, with a different gradient for the different conditions. To test this model we fitted a set of straight lines through the data with a zero intercept. These are shown as the broad lines running through the sets of points in Figure 4. Note that the vertical bars represent one standard error and that the true mean should lie outside of the range of two standard errors approximately five percent of the time. This very simple model appears to be a reasonable first approximation to the data, although as the errors approach $40 \%$ there appears to be some flattening of the curves.

On the basis of these results we conclude that the graph that can be understood with head coupled stereo is about
3.0 times as large as the 2D graph for any given error rate (taking the ratios of the gradients). Using stereo alone appears to increase the comprehensible graph size by approximately a factor of 1.6 and using head coupling alone appears to increase the comprehensible graph size by a factor of 2.2 .

Many visual languages are networks of nodes connected by arcs. Because of the advantage of $3 D$ viewing over $2 D$ viewing, we can confidently predict that as high performance 3D graphics systems become commonplace, many visual languages will evolve from a $2 \mathrm{D}$ to a $3 \mathrm{D}$ layout. However, many challenging design problems will have to be solved in order to create symbology that works well in 3D.

1. Arthur, K., Booth, K.S., and Ware, C. Evaluating Performance in Fish Tank Virtual Reality. ACM Transactions on Information Systrems. 11(3) 216-266.

2. Deering, M. (1992) High resolution virtual reality. Computer Graphics, 26,2,195-202.

3. Sollenberger, R.L and Milgram, P. (1993) The effects of Stereoscopic and Rotational Displays in a ThreeDimensional Path-Tracing Task. Human Factors, 35(3) 483-500.

4. Ware, C., Arthur., and Booth, K.S. (1993)Fish Tank Virtual Reality. Proceedings of INTERCHI'93. 37-42.

5. Ware, C. and Franck, G. (1994) Visualizing Information Nets in Three Dimensions. Technical Report, TR94-082, University of New Brunswick.
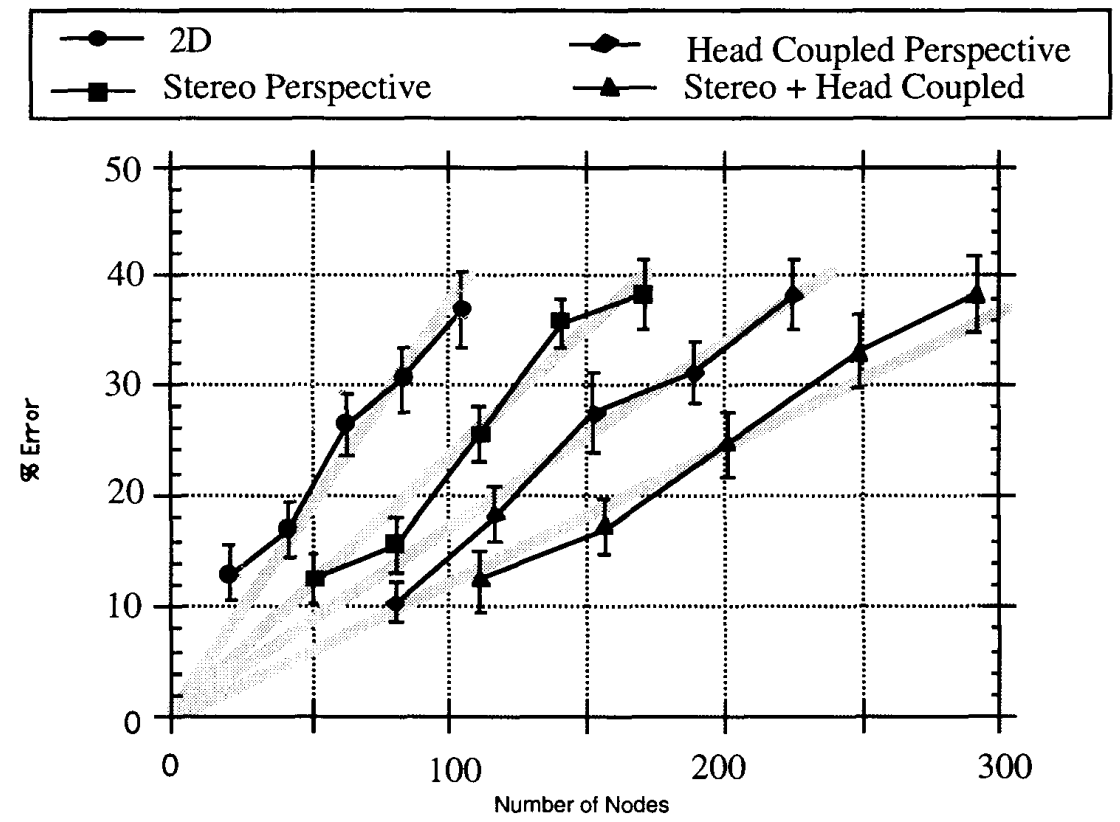

Figure 1. Error data from Experiments 1a and 1b. Vertical bars represent one standard error of the mean. The straight lines represent the simple model described in the text. 